\title{
PUMA-mediated epithelial cell apoptosis promotes Helicobacter pylori infection-mediated gastritis
}

\author{
Yini Dang $\mathbb{D}^{1}$, Yifeng Zhang ${ }^{2}$, Lingyan $\mathrm{Xu}^{3}$, Xiaoying Zhou', Yanhong $\mathrm{Gu}^{3}$, Jian Yu $\mathbb{D}^{4}$, Shidai Jin ${ }^{3}$, Haoming Ji ${ }^{5}$, \\ Yongqian Shu ${ }^{3}$, Guoxin Zhang ${ }^{1}$, Shiyun Cui $\mathbb{0}^{3}$ and Jing Sun ${ }^{3}$
}

\begin{abstract}
The molecular mechanism responsible for Helicobacter pylori infection-mediated gastritis and carcinogenesis is not yet clear. Increased evidence suggests that chronic gastritis and elevated gastric epithelial cell (GEC) apoptosis are crucial events during stomach carcinoma transformation. PUMA is a potent proapoptotic BCl-2 protein and mediates acute tissue injury. In this study, we aimed to investigate the role of PUMA in GEC apoptosis and inflammation induced by $H$. pylori infection. As a result, we found that PUMA expression was elevated in gastritis tissues compared with uninvolved tissues, and it was correlated with the severity of apoptosis and gastritis. In mice, PUMA mRNA and protein were markedly induced in GECS upon induction of gastritis by H. pylori. PUMA-deficient mice were highly resistant to apoptosis and gastritis induced by H. pylori. Furthermore, the transcription factor NF-KB p65 binds to PUMA promoter to activate PUMA transcription after $\mathrm{H}$. pylori infection. In addition, NF-kB inhibitor could rescue $H$. pylori-induced apoptosis and gastritis. Finally, H. pylori-induced activation of p-p65 and PUMA was mediated via Toll-like receptor 2 (TLR2) and blocked in TLR2 knockout mice. Taken together, these results verified the pro-inflammatory effect of PUMA in H. pylori-infected gastric tissue. Moreover, TLR2/NF-KB-mediated transcriptional regulation of PUMA contributes to the pathogenesis of $H$. pylori-infected gastritis.
\end{abstract}

\section{Introduction}

Inflammation is considered a hallmark of cancer, and chronic inflammation plays an essential role in the development of several types of solid tumors. Infection with Helicobacter pylori and the resulting chronic inflammation might be the initial step in stomach carcinogenesis. Once acquired, infection can persist and lead to elevated gastric epithelial cell (GEC) apoptosis and chronic gastritis that can progress to gastric atrophy, metaplasia, and finally gastric carcinoma ${ }^{1,2}$.

\footnotetext{
Correspondence: Guoxin Zhang (guoxinz@njmu.edu.cn) or

Shiyun Cui (cuishiyun@njmu.edu.cn) or Jing Sun (sunj@njmu.edu.cn)

'Department of Gastroenterology, The First Affiliated Hospital of Nanjing Medical University, Nanjing 210029, China

${ }^{2}$ Department of Gastroenterology, Nanjing First Hospital, Nanjing Medical University, Nanjing 210001, China

Full list of author information is available at the end of the article.

These authors contributed equally: Yini Dang, Yifeng Zhang

Edited by. I Amelio
}

Inflammatory responses induced by $H$. pylori infection play a pivotal role in human chronic gastritis ${ }^{3-5}$, partly by activating a complex network of immune signaling, including the nuclear factor (NF)- $\mathrm{kB}$ pathway. Cytotoxinassociated gene A (CagA), lipopolysaccharide (LPS), and peptidoglycan are known virulence factors that significantly contribute to $H$. pylori-induced activation of NF- $\mathrm{KB}$ and its target genes ${ }^{6,7}$ as well as chronic inflammation $^{4,6,8-10}$. However, the key downstream NF-kBdependent targets in GEC apoptosis and chronic inflammatory responses remain to be identified in $H$. pylori infection-mediated carcinogenesis.

p53 upregulated modulator of apoptosis (PUMA) is a BH3-only Bcl-2 family member ${ }^{11,12}$ and functions as a critical initiator of apoptosis in p53-dependent and -independent manner ${ }^{13}$. PUMA potently induces mitochondrial permeabilization, cytochrome $\mathrm{C}$ release, and apoptosis by binding to other Bcl-2 family members, such as $\mathrm{Bax}, \mathrm{Bcl}-2$, and $\mathrm{Bcl}-\mathrm{X}_{\mathrm{L}}{ }^{14-16}$. We have previously

\section{(c) The Author(s) 2020}

(c) (i) Open Access This article is licensed under a Creative Commons Attribution 4.0 International License, which permits use, sharing, adaptation, distribution and reproduction cc) in any medium or format, as long as you give appropriate credit to the original author(s) and the source, provide a link to the Creative Commons license, and indicate if changes were made. The images or other third party material in this article are included in the article's Creative Commons license, unless indicated otherwise in a credit line to the material. If material is not included in the article's Creative Commons license and your intended use is not permitted by statutory regulation or exceeds the permitted use, you will need to obtain permission directly from the copyright holder. To view a copy of this license, visit http://creativecommons.org/licenses/by/4.0/. 
reported that PUMA is directly activated by p65 through the canonical NF- $\mathrm{kB}$ pathway during colonic inflammation in both humans and mice ${ }^{17}$, and it mediates inflammation as well as tumor necrosis factor (TNF)- $\alpha-$ induced intestinal epithelial cell apoptosis ${ }^{18,19}$, suggesting a potential role of PUMA in gastrointestinal inflammation and tissue injury.

We hypothesized that PUMA might be involved in the pathogenesis of gastric cancer by mediating GEC apoptosis induced by $H$. pylori and contribute to chronic gastritis. In this study, we found that PUMA is induced by H. pylori by Toll-like receptor 2 (TLR2)/NF-KB-mediated transcriptional regulation and contributes to GEC apoptosis, gastritis, and the progression of gastric cancer, which is significantly attenuated by genetic ablation of PUMA or TLR2.

\section{Results}

Elevated PUMA expression and apoptosis in $\mathrm{H}$. pyloripositive human gastric tissues

To study the role of PUMA in H. pylori infectionmediated carcinogenesis, we first analyzed 20 pairs of matched $H$. pylori-positive human gastritis tissues with uninvolved tissues. PUMA was found to be elevated in the gastritis tissues compared with uninvolved tissues using immunohistochemistry (IHC) and immunofluorescence (IF) staining (Fig. 1a-c). Quantitation by real-time PCR revealed a nearly fourfold increase in mRNA level of PUMA in gastritis tissue compared with uninvolved tissues (Fig. S1A), which was further confirmed by western blotting (Fig. 1d). In addition, PUMA expression was found to be significantly correlated with the severity of gastritis, which was marked by elevated apoptosis compared with the uninvolved tissues using terminal deoxynucleotidyl transferase-mediated dUTP-fluorescein nick end labeling (TUNEL) staining (Figs. 1e, $\mathrm{f}$ and S1B). Western blot of caspase3 and cleaved-caspase 3 and IHC analysis of cleaved-caspase 3 also verified the correlation between PUMA expression and elevated apoptosis (Fig. S1C, D). In addition, western blot analysis of PUMA expression revealed increased induction of PUMA in most H. pylori-positive gastric cancer tissues compared with the negative controls (Fig. S1E). The above observations prompted us to further investigate the role of PUMA in gastritis using human cell lines and mouse models. Upon incubation with $H$. pylori at a ratio of 100:1 (bacteria to cell), we found that PUMA mRNA levels were increased by eightfold within $24 \mathrm{~h}$ in the human GEC line AGS (Fig. $1 \mathrm{~g})$. In addition, we used mice infected with $H$. pylori to determine PUMA expression. Western blot and IHC analysis were performed at $24 \mathrm{~h}, 48 \mathrm{~h}$, and 7 days after $H$. pylori treatment. As shown in Figs. 1h and S1F, PUMA protein was induced after $\mathrm{H}$. pylori treatment and elevated from $24 \mathrm{~h}, 48 \mathrm{~h}$ to 7 days. Collectively, we reasoned that
PUMA might play roles in $H$. pylori-induced gastritis and gastric cancer, while $H$. pylori infection can lead to rapid induction of PUMA in GECs.

\section{PUMA induced apoptosis in an $H$. pylori-treated gastric cancer cell line}

PUMA is a downstream target of p53 and plays a critical role in mediating both p53-dependent and -independent apoptosis $^{20}$. To probe the potential role of p53 in PUMA induction, gastric cancer cell lines with different p53 status were treated with $H$. pylori for $60 \mathrm{~h}$. The expression of p53 in both p53-wild type (p53-WT) and -mutant cell lines was elevated after $H$. pylori treatment (Fig. S2A). While PUMA expression was also elevated in both p53-WT and -mutant cell lines, it suggested p53independent activation of PUMA (Fig. 2a). We further generated PUMA-KO AGS cells using CRISPR to determine whether $P U M A$ plays a role in apoptosis following H. pylori infection (Fig. S2B). The success of PUMA knockout in AGS cell lines was confirmed by DNA sequencing and western blot (Fig. S2C). Using flow cytometry, we found a significantly smaller proportion of apoptotic cells in PUMA knockout (KO) cells by $60 \mathrm{~h}$ (Fig. $2 \mathrm{~b}, \mathrm{c}$ ), with increased cell viability, compared with the control groups. Furthermore, AGS cells were infected with a PUMA-adenovirus or control for $24 \mathrm{~h}$ and then treated with $H$. pylori for $60 \mathrm{~h}$ (Figs. 2b and S2D). The proportion of apoptotic cells in Ad-PUMA-infected cells was not significantly increased compared with the control groups (Fig. 2c). Western blot analysis indicated reduced activation of caspase3 and caspase8 in PUMA-KO AGS cells compared with WT cells. Consistent with the flow cytometric analysis, we observed a decreased cleavedcaspase3 and caspase8 in PUMA-KO AGS cells (Fig. 2d). In addition, we used CCK8 to detect apoptotic cells after H. pylori treatment and similar results were found (Fig. 2e). These results indicated that PUMA plays a critical role in cell apoptosis induced by $H$. pylori.

\section{PUMA-deficient mice are resistant to $H$. pylori-induced gastritis apoptosis}

To determine whether the induction of PUMA contributes to $H$. pylori-induced chronic gastritis in vivo, we compared the pathological state and apoptosis using WT and PUMA-deficient (PUMA-KO) mice. Two months after infection, all mice were sacrificed and the infection status was verified by Giemsa staining and rapid urease test (Fig. S3A, B). Hematoxylin-eosin (H\&E) staining revealed that submucosal inflammation was significantly blunted in PUMA-KO mice after $H$. pylori treatment for 2 months (Fig. 3a). Apoptosis was detected by TUNEL staining and western blot of caspase 3 and cleavedcaspase 3 as well as IHC analysis of cleaved-caspase 3 in the gastric mucosa after $H$. pylori treatment for 2 months. 


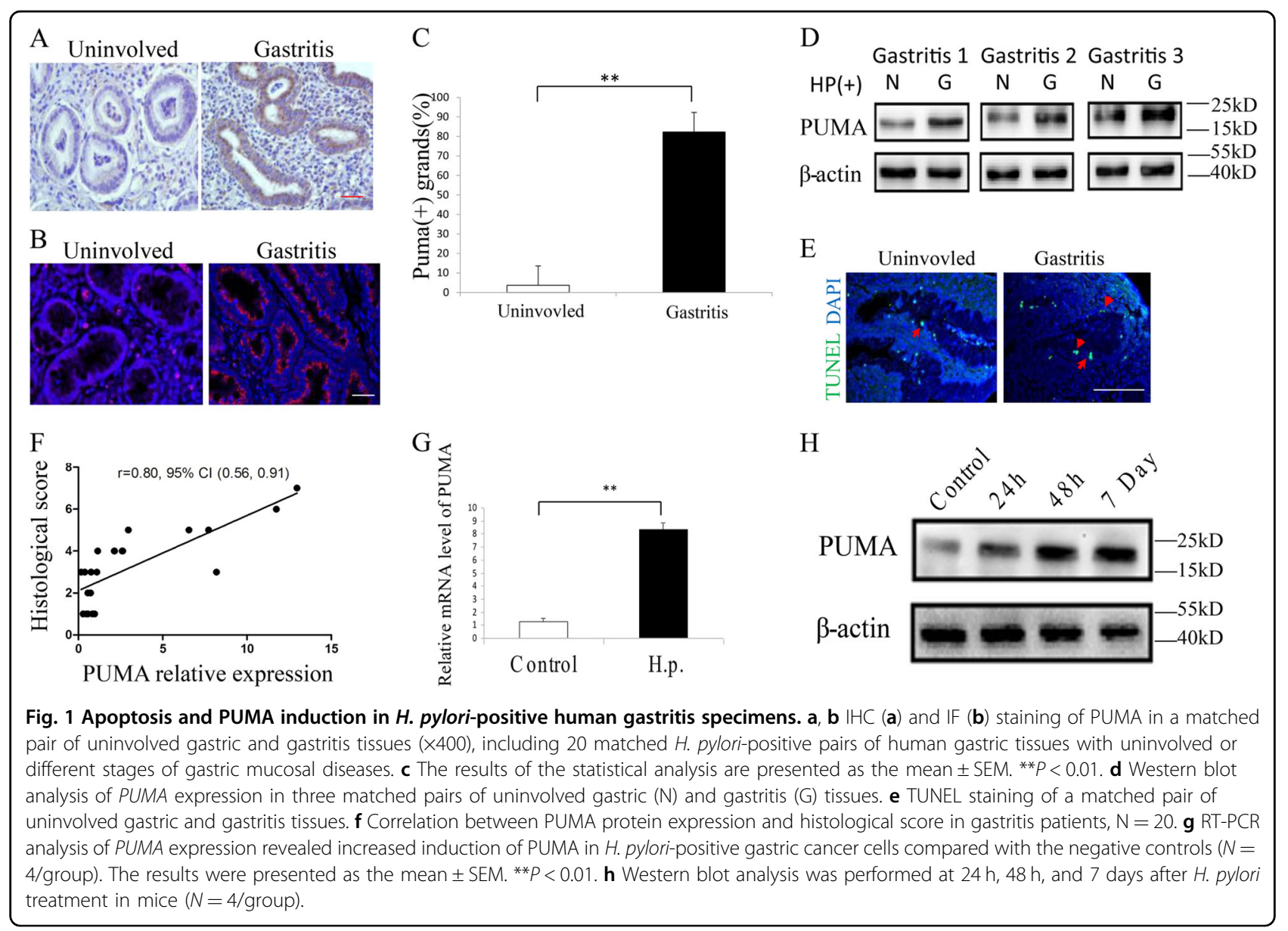

Apoptosis markedly increased in WT mice, which was blocked in PUMA-KO mice (Figs. 3b-d and S3C, D). Interestingly, we also found that the expression of intrinsic factor, a glycoprotein that is essential for the absorption of cobalamin, and TFF1 (trefoil factor 1), which is mainly expressed in the fundus and antrum of gastric mucous cells ${ }^{21}$, increased in PUMA-KO mice (Figs. 3e, f). These results confirm the critical role of PUMA in GEC apoptosis and gastritis.

\section{p65 binds to the PUMA promoter to activate PUMA transcription following $H$. pylori treatment}

We then explored whether p53-independent activation of PUMA is mediated by the canonical NF- $\mathrm{KB}$ pathway upon $H$. pylori-induced treatment. In the NF- $\mathrm{KB}$ signaling cascade, phosphorylation of p65 is required for nuclear translocation and transcriptional activation ${ }^{22}$. Thus we detected time-dependent increases in p-p65 (S536) and PUMA in AGS cells upon $H$. pylori treatment (Fig. 4a). Pretreatment with the NF- $\mathrm{kB}$ inhibitor BAY 11-7082 for $1 \mathrm{~h}$ blocked p65 activation (p-p65) and PUMA induction by $H$. pylori or TNF- $\alpha$ at $24 \mathrm{~h}$ (Fig. $4 \mathrm{~b}$ ). Transfecting cells

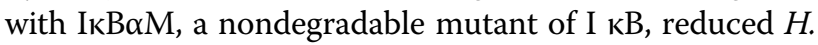

pylori-induced PUMA expression and p65 phosphorylation in WT AGS cells (Fig. 4c), indicating that $H$. pyloriinduced p65 activation is mediated by $\mathrm{I} \kappa \mathrm{B}$ depletion through the canonical NF- $\mathrm{kB}$ pathway. Knockdown of $\mathrm{p} 65$ using small interfering RNA (siRNA) completely inhibited PUMA induction at $60 \mathrm{~h}$ (Fig. 4d). Using reporter assays ${ }^{19}$, we found that fragments $\mathrm{A}, \mathrm{E}$ (the proximal 495-bp region of the PUMA promoter), and $\mathrm{D}$ (the NF- $\mathrm{kB}$ responsive element distal region) were activated by p65 (Fig. 4e). Treatment of mice with the NF-kB inhibitor Bay11-7082 for 3 days $(8 \mathrm{mg} / \mathrm{kg} /$ day $)$ significantly inhibited PUMA induction (Fig. 5a) and apoptosis (Fig. 5b-d and S4A, B) in gastric tissues. These findings suggest that p65 directly activates PUMA transcription through binding to the distal site of PUMA promoter upon H. pylori treatment to induce GEC apoptosis.

\section{H. pylori treatment-induced activation of PUMA and gastritis is blocked by TLR2-KO}

TLRs are pattern recognition receptors that are crucial for sensing pathogens, including $H$. pylori, and subsequent activation of the host immune response and NF$\kappa B$ signaling ${ }^{23}$. We further investigated whether TLR2, 


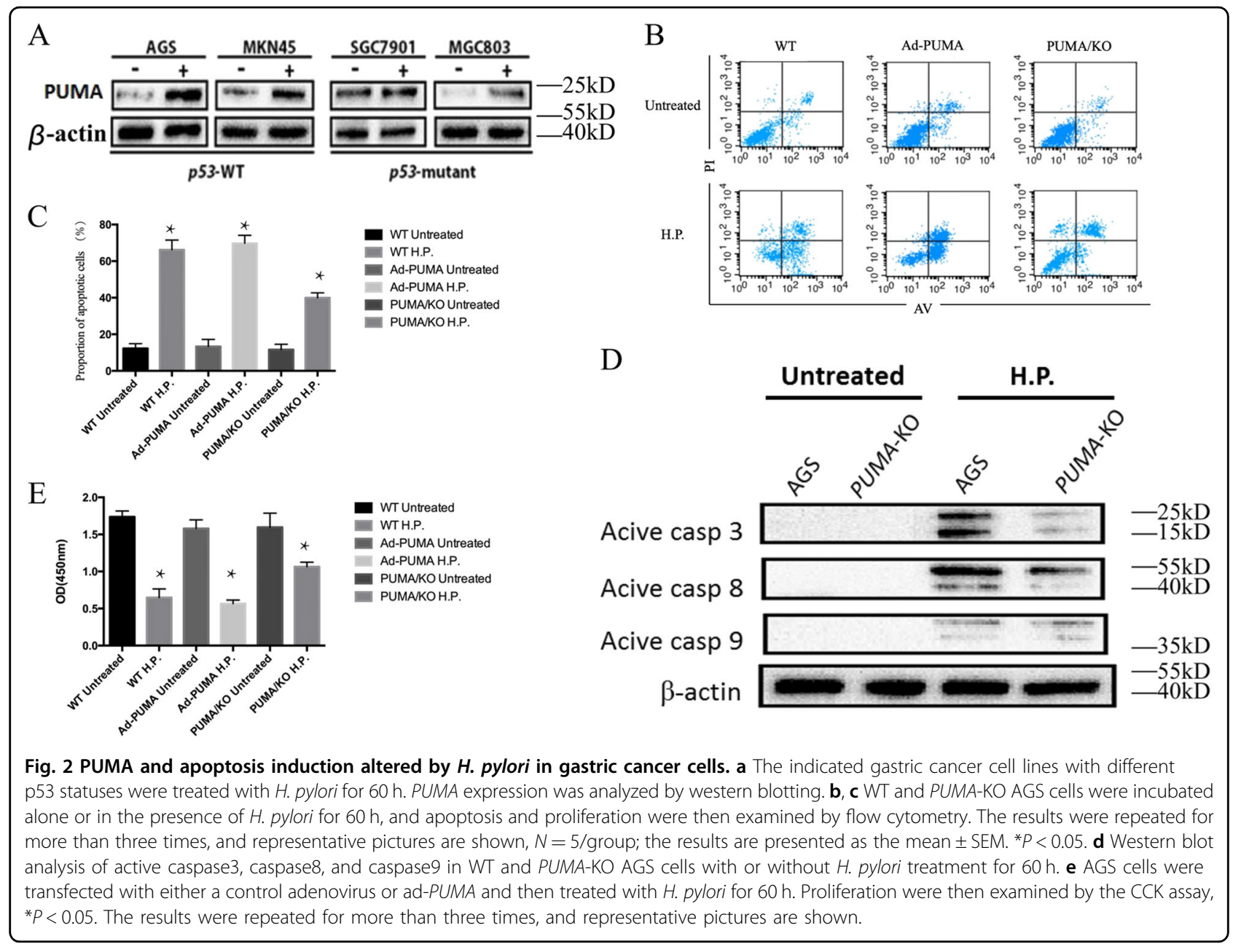

which was previously implicated in the $H$. pylori-induced response, could mediate the activation of $p-p 65$ and PUMA in H. pylori-induced gastritis. Knockdown of TLR2 by siRNA in AGS cells strongly decreased $H$. pyloriinduced $p$ - $p 65$ and PUMA expression (Fig. 6a). Furthermore, we used TLR2-KO mice to explore the role of TLR2 in $H$. pylori-induced gastritis. H\&E staining revealed that submucosal inflammation was induced by $H$. pylori treatment in WT mice, but it was significantly blunted in TLR2-KO mice (Fig. 6b). TUNEL staining (green) revealed a tenfold decrease in GEC apoptosis in TLR2-KO compared with WT mice (Fig. 6c, d). The result was also verified by western blot analysis of caspase 3 and cleavedcaspase 3 and IHC staining of cleaved-caspase3 (Fig. S5A, $\mathrm{B})$. IHC analysis further confirmed the reduced activation of PUMA in the TLR2-KO mice after H. pylori treatment (Fig. 6e). In addition, western blotting revealed that p-p65 and PUMA was induced by 2 months of $H$. pylori treatment in WT mice, but it was significantly blunted in TLR2-KO mice (Fig. S5C). These results suggest that TLRs likely play a key role in directly regulating GEC cell death during innate immune responses in acute inflammation.

\section{Discussion}

The inflammatory response to $H$. pylori infection can initiate and accelerate a sequence of oncogenic events characterized by damage to the gastric epithelium, elevated apoptosis, and inappropriate cellular proliferation, which renders the cell vulnerable to further neoplastic changes $^{24}$. Elevated apoptosis has been demonstrated to be associated with $H$. pylori-positive gastritis, while apoptotic cells are rare in the neck region of the gastric glands (the regenerative cell zone) in normal gastric mucosa. Following initial H. pylori infection, atrophic gastritis progresses, the regenerative cell zone shifts downward, and a relatively large number of apoptotic cells are observed $^{25}$. However, the precise mechanisms of $H$. pylori-induced gastric cancer and GEC apoptosis have not been completely illustrated. In this study, we found that increased PUMA expression was correlated with the level of apoptosis, severity of human gastritis, and gastric 


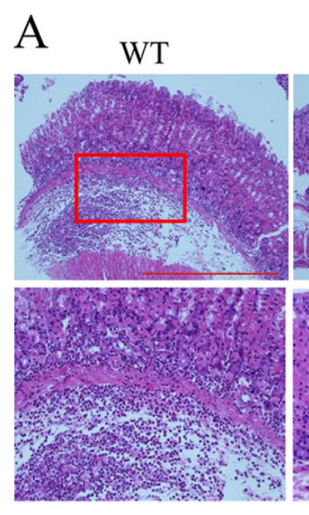

B

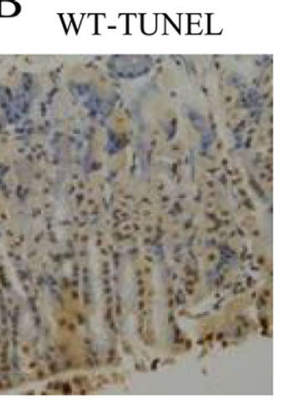

$\mathrm{E}$

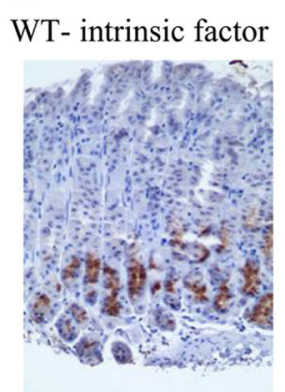

PUMAKO

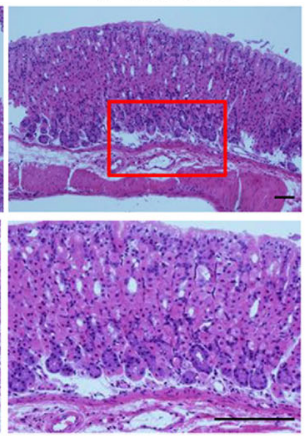

PUMA-KO-TUNEL

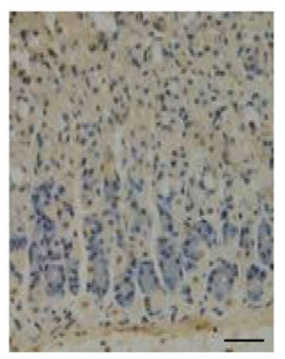

$\mathrm{C}$

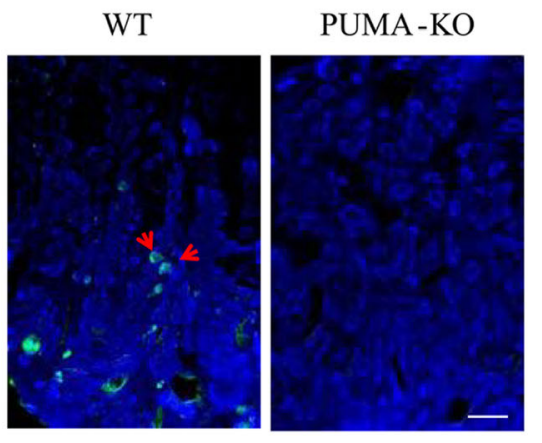

$\mathrm{D}$

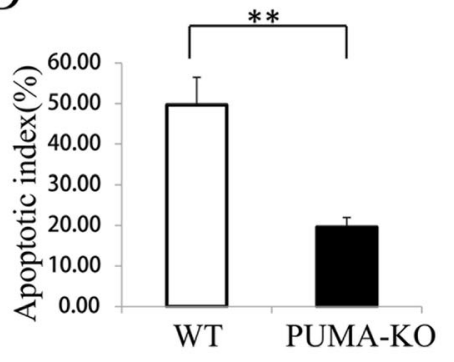

Fig. 3 Suppression of $H$. pylori infection-mediated carcinogenesis, apoptosis, and differentiation in PUMA-deficient mice (WT and PUMAKO mice were treated with $H$. pylori for 2 months to induce gastritis). a H\&E staining of gastric tissues from WT and PUMA-KO mice after $H$. pylori treatment for 2 months $(\times 100)$. Boxed regions of the glands are magnified in the lower panel $(\times 200)$. b TUNEL IHC staining of gastric tissues from the treated mice $(\times 200)$. c TUNEL (green) IF staining of gastric tissues from the treated mice after $\mathrm{H}$. pylori treatment for 2 months, $N=5 / g r o u p ;$ the results are presented as the mean \pm SEM. $\mathbf{d}$ The apoptosis index was measured by counting TUNEL signals in 100 randomly selected glands.

${ }^{*} P<0.01$. e Staining for intrinsic factor showed mature zymogenic cells in $\mathrm{H}$. pylori-treated wild type (left) and PUMA-KO (right) mice for 2 months. f Staining for TFF1 showed surface mucous cells in wild type (left) and PUMA-KO (right) mice treated with H. pylori for 2 months.

cancer. PUMA deficiency in mice abrogated H. pyloriinduced gastritis and GEC apoptosis, supporting the function of PUMA as a critical mediator of GEC apoptosis and a significant modulator of $H$. pylori-related gastritis. PUMA is necessary for TNF- $\alpha$-induced apoptosis through intrinsic pathways ${ }^{19}$. Consistent with previous studies, we found that the activation of PUMA by NF- $\mathrm{kB}$ promoted caspase activation $^{26-28}$.

Inflammatory conditions are characterized by activation of the transcription factor NF- $\mathrm{kB}$, resulting in the expression of NF-kB-regulated, inflammation-related genes, such as inducible nitric oxide synthase and cyclooxygenase-2. In this study, we identified PUMA as a downstream target of NF-kB and a critical mediator of $H$. pylori-induced GEC apoptosis and gastric cancer. PUMA mRNA and protein were consistently activated in cells treated with both $H$. pylori and TNF- $\alpha$. The induction of PUMA by $H$. pylori required the p65 subunit of NF- $k B$ and was mediated by a ІкB site located in the distal region of PUMA promoter. Our results showed that a deficiency in the p65 component of NF- $\mathrm{BB}$, a key regulator of inflammation ${ }^{17}$, blunted PUMA induction in vitro and 

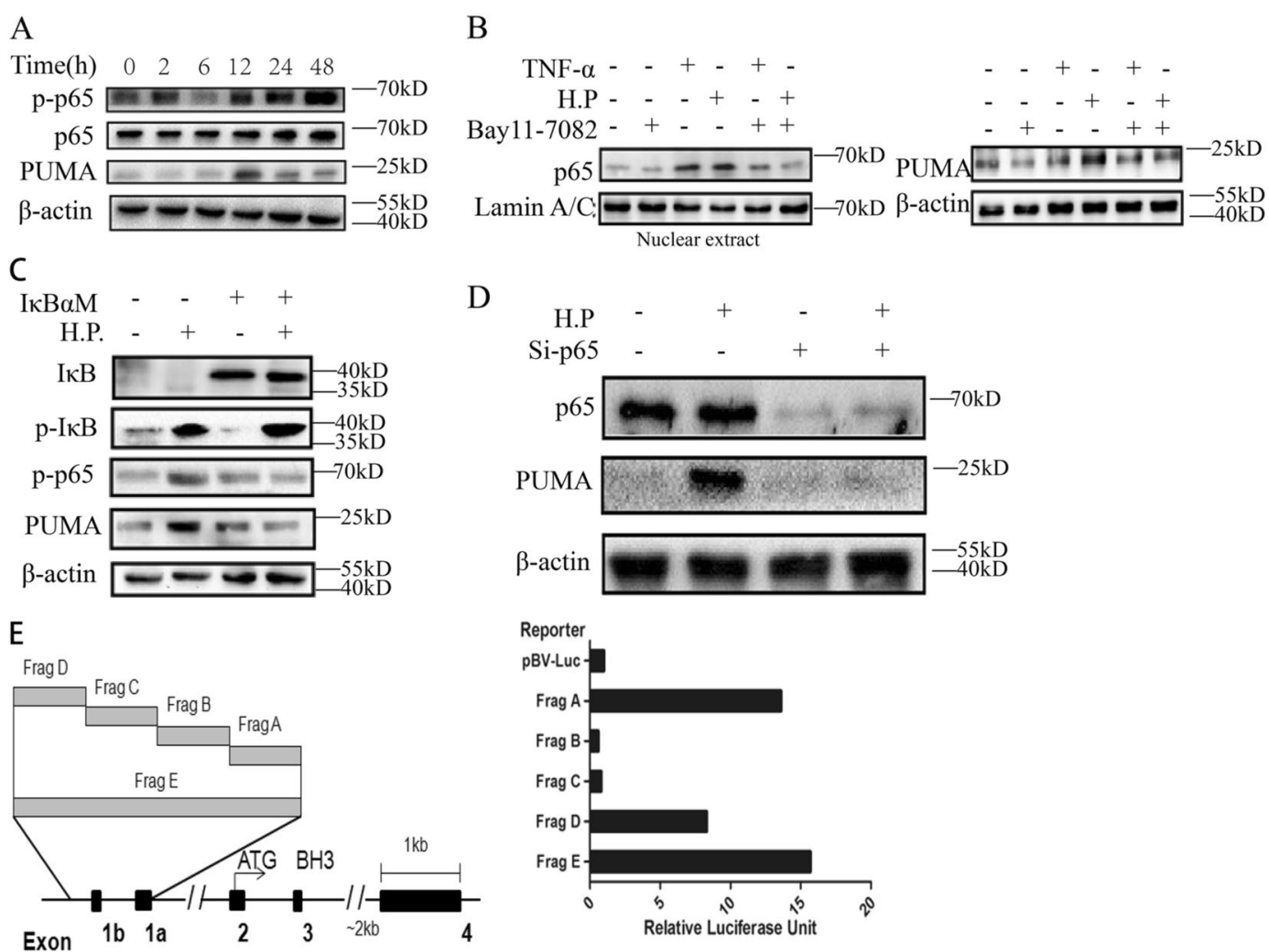

D
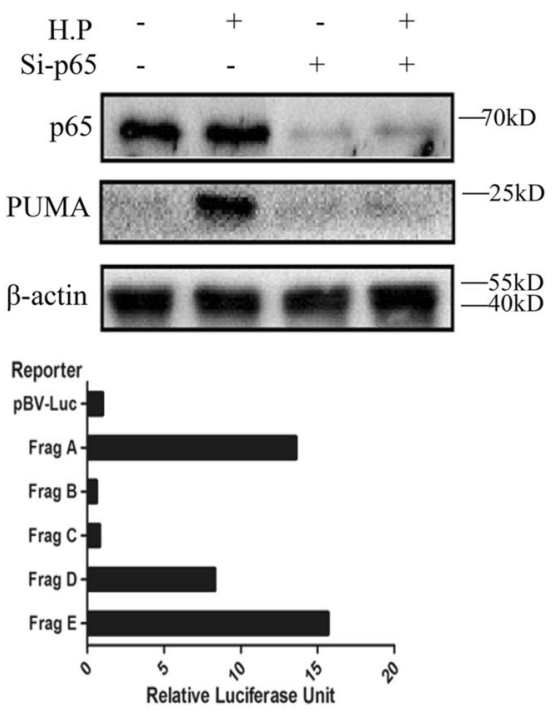

Fig. 4 P65 directly binds to the PUMA promoter to activate its transcription following H. pylori treatment. a Expression of $p$-p65 (S536), p65, and PUMA on AGS cells after treatment with $\mathrm{H}$. pylori at the indicated time points was analyzed by western blotting. $\mathbf{b}$ AGS cells were treated with $10 \mathrm{mmol} / \mathrm{L}$ BAY $11-7082$ for $1 \mathrm{~h}$ and then with H. pylori or $10 \mathrm{ng} / \mathrm{mL}$ TNF-a for $24 \mathrm{~h}$. Left, nuclear fractions were isolated from cells and analyzed for p65 expression by western blotting; right, western blot analysis of PUMA and $\beta$-actin expression in whole-cell lysates after $H$. pylori or TNF-a treatment. cWT AGS cells were transfected overnight with $\mathrm{PCMV}$ or IKBaM and then treated with $H$. pylori for $24 \mathrm{~h}$. The expression levels of PUMA, $p$-IKB, IKB, and $p$ - 565 were analyzed by western blotting. $\mathbf{d}$ AGS cells were transfected with either a control scrambled siRNA or a p65 siRNA for $24 \mathrm{~h}$ and then treated with H. pylori for 60 h. p65 and PUMA expression was probed by western blotting. e Left, schematic representation of the genomic structure of PUMA highlighting the PUMA promoter fragments (Frag) A-E of the PUMA promoter, followed by treatment with $\mathrm{H}$. pylori. Reporter activities were measured $24 \mathrm{~h}$ later by a luciferase assay.

in vivo. This observation, along with the finding that NF$\kappa \mathrm{B}$ or TNF- $\alpha$ was activated in $H$. pylori-treated mice, suggested that NF- $\mathrm{kB} / \mathrm{TNF}-\alpha$ were responsible for PUMA induction and subsequent GEC apoptosis. These results established the direct regulation of a $\mathrm{BH} 3$-only $\mathrm{Bcl}-2$ family member by NF- $\mathrm{KB}$ during acute gastritis via a p53independent mechanism.

Several products are currently thought to be important for the activation of NF- $\mathrm{BB}$ by $H$. pylori: LPS, peptidoglycan, and $\operatorname{CagA}^{29,30}$. LPS has been shown to be recognized by host cells by binding to either TLR2 or TLR4 and has a role in H. pylori-induced NF- $\mathrm{KB}$ activation and the inflammatory response ${ }^{23,31}$. In macrophages, H. pylori activates NF-KB via TLR2 (for induction of interleukin (IL)- 6 and IL-1 $\beta$ ) and TLR4 (for induction of IL-12, IL-10, and IL-8) $)^{23}$. In our study, $p$-p65 and PUMA were decreased after TLR2 knockdown in the AGS cell line. In addition, H\&E/IF staining revealed that the TLR2-
KO mice were highly resistant to $H$. pylori-induced gastritis due to blocked NF- $\mathrm{KB}$ activation and PUMA induction. Our novel findings suggest that GEC death and PUMA induction are likely triggered by TLR2-mediated activation of NF- $\mathrm{kB}$ and amplified via subsequent induction of inflammatory cytokines. Further studies are needed to investigate which of these interconnected nodes in the signaling pathways might be pharmacologically modulated to improve epithelial healing and resolution of the immune response to prevent chronic gastritis and associated cancer.

In summary, our results demonstrate that TLR2/NF$\kappa \mathrm{B}$-mediated PUMA induction contributes to the pathogenesis of $H$. pylori-induced gastritis by promoting GEC apoptosis. Inhibition of PUMA directly using a small molecular inhibitor ${ }^{32}$ or indirectly using anti-TLR2 ${ }^{33}$ might represent a novel approach to reduce GEC death and to prevent the development of chronic gastritis. 

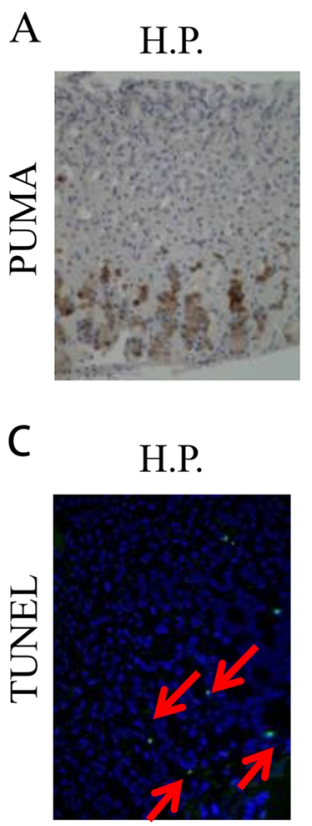

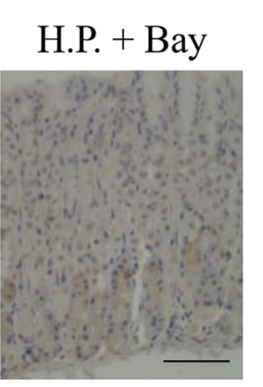

H.P. + Bay

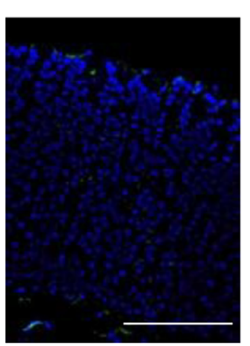

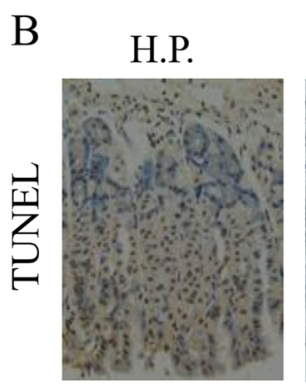

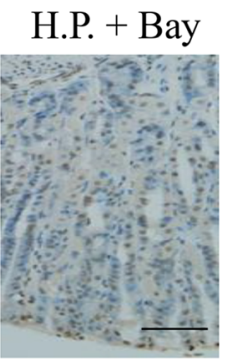

$\mathrm{D}$

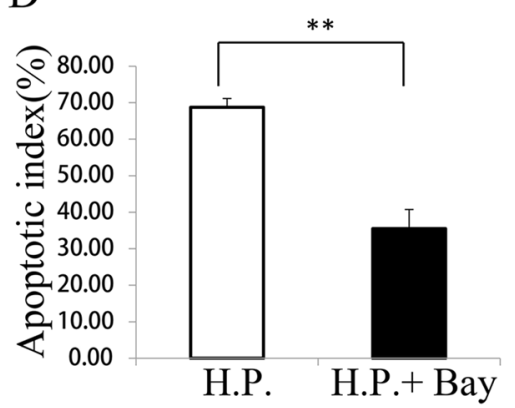

Fig. 5 Effects of NF-KB inhibition on $H$. pylori-induced apoptosis and PUMA expression (WT mice were treated with $H$. pylori alone or in combination with $8 \mathrm{mg} / \mathbf{k g}$ of the NF- KB inhibitor Bay $\mathbf{1 1 7 0 8 2}$ for 3 days). a IHC staining of PUMA in gastric tissues from the treated mice. b TUNEL (brown) staining of gastric tissues from the treated mice. c TUNEL (green) IF staining of gastric tissues from the treated mice. $\mathbf{d}$ The apoptotic index was calculated by counting TUNEL signals in 100 randomly selected glands following TUNEL staining, as in c. $N=4 / g r o u p$, the results are presented as the mean $\pm S E M,{ }^{* *} P<0.01$ compared with the control.

\section{Materials and methods Tissue samples}

The acquisition for all human tissues was approved by the Institutional Review Board of the Digestive Department of the First Affiliated Hospital of Nanjing Medical University. All subjects included 20 frozen pairs of matched $H$. pylori-positive gastritis and 20 uninvolved tissues recruited from the First Affiliated Hospital of Nanjing Medical University in 2017. H. pylori detection was confirmed by ${ }^{13} \mathrm{C}$ breath test. Among the 20 patients in the gastritis group, gastritis tissue was found in the antrum of 6 patients, fundus of 3 patients, corpus of 7 patients, and cardia of 4 patients. Thirteen males and 7 females represented in the $H$. pylori-positive group, ranging in age from 37 to 69 years, whereas 14 males and 6 females comprised the uninvolved group, ranging in age from 24 to 73 years. Among the 20 patients in the gastritis group, none had received prior treatment. Cancer subjects included three $H$. pylori-positive gastric cancer and three negative gastric cancer tissues. All the sample studies have obtained patient consent and were collected by gastroscopy biopsy. Both of the tissues from human and mice were randomly selected and the investigators were blinded to the group allocation during the experiment.

\section{Cell culture and treatment}

The gastric cancer cell lines were cultured in RPMI 1640 (Gibco) supplemented with $10 \%$ fetal bovine serum. All the cells were maintained at $37{ }^{\circ} \mathrm{C}$ with $5 \% \mathrm{CO}_{2}$. The source and mycoplasma contamination of the cell lines were evaluated by Beijing YueWei Gene Technology Co., Ltd in October, 2013: DNA prepared from our cells using a commercial Chelex100 kit was analyzed by STR (Short tandem repeat) profiling. Cell lines were considered to be identical to the ATCC corresponding cell lines when the entered short tandem repeat (STR) profiles yield 100\% match to the ATCC STR database. No cross-contaminated cell lines or mycoplasma contamination was detected. All the in vitro experiments were repeated three times. H. pylori bacteria was grown on Columbia agar plates (bioMérieux, Marcy) with selective supplement (Oxoid, Basingstoke, UK) under microaerophilic conditions using an anaerobic chamber (BBL Campy Pouch System, Becton Dickinson Microbiology Systems, San Diego, CA, USA) at $37^{\circ} \mathrm{C}$ for 48-72 h. H. pylori bacteria utilized for all experiments is the cytotoxic $(\mathrm{CagA}+/ \mathrm{VacA}+)$ reference strain of $H$. pylori SS1 $(\mathrm{ATCC})^{34}$. The densities of the bacteria were measured by the optical density (OD) at $660 \mathrm{~nm}\left[1\right.$ OD660 $=1$ dens $^{8}$ colony-forming units (CFU)/ml]. For $H$. pylori treatment, AGS cells were infected with the bacteria at a cell-to- 

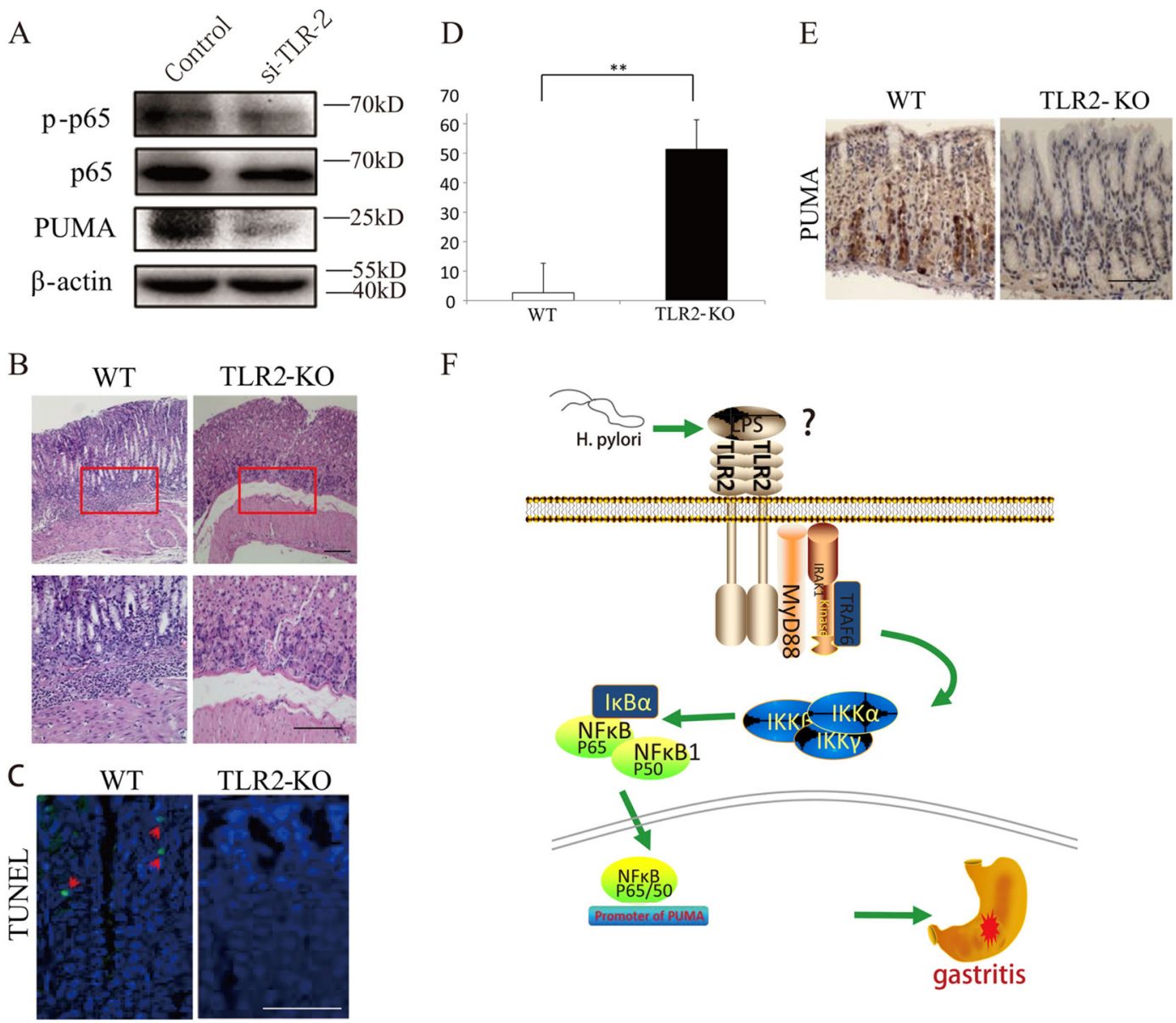

F
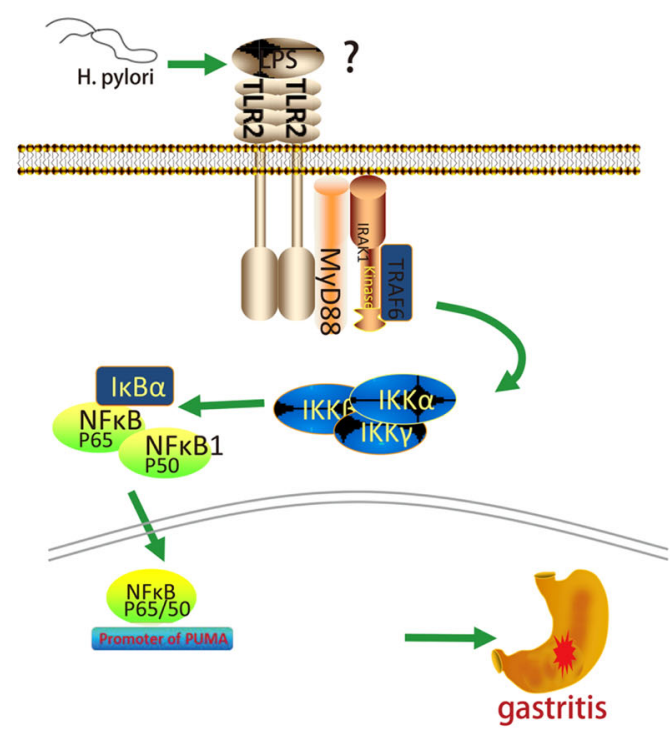

Fig. 6 TLR 2 inhibition can suppress H. pylori-induced gastritis by downregulating PUMA (WT and TLR2-KO mice were treated with H. pylori for $\mathbf{2}$ months to induce gastritis, $\boldsymbol{N}=\mathbf{5}$ for each group). a AGS cells were transfected with either a control scrambled siRNA or a TLR2 siRNA for $48 \mathrm{~h}$ and then treated with H. pylori for $24 \mathrm{~h} . \mathrm{p}$-p 65 and PUMA expression were probed by western blotting. $\mathbf{b}$ H\&E staining of gastric tissues from WT and TLR2-KO mice after $H$. pylori treatment for 2 months $(\times 100)$. Boxed regions of the glands were magnified in the lower panel $(\times 200)$. c, $\mathbf{d}$ Representative images of TUNEL staining (green) of gastric tissues from treated mice. WT and TLR2-KO mice were treated with $H$. pylori for $48 \mathrm{~h}$, $N=5 /$ group; the results are presented as the mean \pm SEM. ${ }^{* *} P<0.01$. e Representative images of PUMA IHC staining of gastric tissues from treated mice. WT and TLR2-KO mice were treated with $\mathrm{H}$. pylori for $48 \mathrm{~h}$. $\mathbf{f}$ Proposed roles of the TLR2 and PUMA involved in H. pylori-induced gastritis.

bacterium ratio of $1: 100$ for the indicated times in the culture medium.

\section{Establishment of PUMA knockout cell line}

To knock PUMA in AGS cell line, we cloned a singleguide RNA (sgPUMA-1: AAACGCGCACGCCAGGAGG GCAGC; sgPUMA-2: CACCGTAGAGGGCCTGGCCCG CGA) into the corresponding pCas9 vector. The constructed plasmid was sequenced and then transfected to AGS cells with Lipofectamine 2000. Later, we extracted the genomic DNA of transfected AGS cells and amplified the PUMA gene. Subsequently, we selected the single colony and confirmed the PUMA knockout AGS cell lines by DNA sequencing and western blot.

\section{Mice and treatment}

The procedures for all animal experiments were approved by the Institutional Animal Care and Use Committee of the First Affiliated Hospital of Nanjing Medical University. Mice on the C57BL/6 background with different genotypes, including WT, PUMA-KO (PUMA-/-), and TLR2-KO (TLR2-/-), were generated by breeding and identified by PCR genotyping as previously described: The PUMA KO mice were originally from G. P. Zambetti and had been crossed to B6 backcrossed to the C57BL/ 6 background for $>10$ generations (F10), and the TLR2-KO mice were originally from Carsten J. Kirschning and had been crossed to 129SV backcrossed to the C57BL/6 background for $>10$ generations 
$(\mathrm{F} 10)^{35-37}$. The mice were housed in microisolator cages and allowed access to water and chow ad libitum. Threeto-4-week-old littermates were treated with $H$. pylori by injection of $0.4 \mathrm{~mL}$ brucella broth containing $3 \times 10^{8} \mathrm{CFU}$ H. pylori using a feeding needle for 7 days to induce gastritis. Negative controls that were used in all experiments showed no significant submucosal inflammation after broth medium treatment for 2 months by H\&E staining (Fig. S5D). Two months after infection, all mice were sacrificed, and the stomach tissues were collected for further analysis. For NF- $\mathrm{kB}$ inhibition, mice were injected $1 \mathrm{~h}$ prior to $H$. pylori treatment with $8 \mathrm{mg} / \mathrm{kg}$ of the NF- $\mathrm{kB}$ inhibitor BAY 117082 (EMD Biosciences) once daily and then sacrificed at the indicated time points.

\section{IHC and IF staining}

The frozen tissues of gastritis cases and uninvolved tissues were used to prepare sections. Tissue sections $(5 \mu \mathrm{m})$ were deparaffinized, rehydrated, and treated with $3 \%$ hydrogen peroxide, followed by antigen retrieval in boiling $0.1 \mathrm{M}$ citrate ( $\mathrm{pH}$ 6.0) buffer once for $10 \mathrm{~min}$. The sections were then blocked with $20 \%$ rabbit serum for $30 \mathrm{~min}$. PUMA staining was performed at $4{ }^{\circ} \mathrm{C}$ overnight using a rabbit anti-PUMA antibody (Prosci 3043) with Alexa 594 (Invitrogen) for signal detection. Cleavedcaspase 3 staining was performed at $4{ }^{\circ} \mathrm{C}$ overnight using a rabbit anti-Caspase 3 antibody (Proteintech 19677-1-AP) for signal detection. Cells with positive staining were scored in at least 100 crypt sections and reported as the mean \pm standard deviation (SD). Every scale indicates $100 \mu \mathrm{m}$.

\section{Analysis of mRNA and protein expression}

Total RNA was extracted using TRIzol Reagent (Invitrogen) and reverse-transcribed using the High Capacity RNA-to-cDNA Kit (Takara). The expression of cDNA was quantified using Taqman Gene Expression Master Mix with an ABI 7900HT System (Applied Biosystems). The primer of PUMA is m-PUMA 3'-AGCAGCACTTAGAGTCGCC and PUMA 5'-CCTGGGTAAGGGGAGGAGT. Western blotting was performed using antibodies against human PUMA, murine PUMA (Abcam), p65 (Santa Cruz), active caspase3 (Cell Signal), phospho-p65 (Ser536) (Cell Signal), Bad (Cell Signaling Technology), and $\beta$-actin (Sigma), as previously described $^{14}$. All the experiments were repeated three times.

\section{Analysis of tissue damage and histology}

Histological analysis of gastric tissue was performed by H\&E staining. Histological scores evaluating chronic inflammation were determined as $0-3$ based on previously described criteria $^{38}: 0=$ normal, $1=$ mild, $2=$ moderate, $3=$ marked.

\section{Analysis of apoptosis by flow cytometry}

The different treated cells were trypsinized and centrifuged at $12,000 \times g$ for $5 \mathrm{~min}$ at $4{ }^{\circ} \mathrm{C}$. The cells were washed in D-Hanks solution at $4{ }^{\circ} \mathrm{C}$, and cell apoptosis was detected using the apoptosis kit (eBioscience, USA).

\section{Statistical analysis}

For the in vitro and in vivo experiments, the data are presented as the mean \pm SEM. Statistical analyses were performed using two-tailed Student's $t$ test for parametric data and Pearson's chi-squared test $\left(\mathrm{x}^{2}\right)$ for categorical data. ${ }^{*} P<0.05$ was considered to be statistically significant, and ${ }^{* *} P<0.01$ was considered to be statistically highly significant.

\section{Acknowledgements}

This study was supported by the National Natural Science Foundation of China (No. 81302109 to J.S. and No. 81502611 to S.C.).

\section{Author details}

'Department of Gastroenterology, The First Affiliated Hospital of Nanjing Medical University, Nanjing 210029, China. ${ }^{2}$ Department of Gastroenterology, Nanjing First Hospital, Nanjing Medical University, Nanjing 210001, China. ${ }^{3}$ Department of Oncology, The First Affiliated Hospital of Nanjing Medical University, Nanjing 210029, China. ${ }^{4}$ Department of Pathology and Radiation Oncology, University of Pittsburgh School of Medicine, Pittsburgh, PA 15213, USA. ${ }^{5}$ Department of Oncology, Haian People's Hospital, Nantong 226630, China

Conflict of interest

The authors declared that they have no conflict of interest.

\section{Publisher's note}

Springer Nature remains neutral with regard to jurisdictional claims in published maps and institutional affiliations.

Supplementary Information accompanies this paper at (https://doi.org/ 10.1038/s41419-020-2339-x).

Received: 27 March 2019 Revised: 8 February 2020 Accepted: 10 February 2020

Published online: 20 February 2020

\section{References}

1. Vale, F. F. \& Vitor, J. M. Transmission pathway of Helicobacter pylori: does food play a role in rural and urban areas? Int. J. Food Microbiol. 138, 1-12 (2010).

2. Correa, P. Human gastric carcinogenesis: a multistep and multifactorial process-First American Cancer Society Award Lecture on Cancer Epidemiology and Prevention. Cancer Res. 52, 6735-6740 (1992).

3. Sokolova, O., Maubach, G. \& Naumann, M. MEKK3 and TAK1 synergize to activate IKK complex in Helicobacter pylori infection. Biochim. Biophys. Acta 1843, 715-724 (2014).

4. Devi, S. et al. Helicobacter pylori antigen HP0986 (TieA) interacts with cultured gastric epithelial cells and induces IL8 secretion via NF-kappaB mediated pathway. Helicobacter 19, 26-36 (2014).

5. Peng, L. S. et al. Elevated interleukin-32 expression is associated with Helicobacter pylori-related gastritis. PLOS ONE 9, e88270 (2014).

6. Kang, D. W. et al. Rebamipide abolishes Helicobacter pylori CagA-induced phospholipase D1 expression via inhibition of NFkappaB and suppresses invasion of gastric cancer cells. Oncogene 32, 3531-3542 (2013).

7. Lamb, A. et al. Helicobacter pylori CagA activates NF-kappaB by targeting TAK1 for TRAF6-mediated Lys 63 ubiquitination. EMBO Rep. 10, 1242-1249 (2009). 
8. Saravanan, S. et al. Swertiamarin attenuates inflammation mediators via modulating NF-kappaB/I kappaB and JAK2/STAT3 transcription factors in adjuvant induced arthritis. Eur. J. Pharmacol. Sci. 56, 70-86 (2014).

9. Kim, G. D., Oh, J., Park, H. J., Bae, K. \& Lee, S. K. Magnolol inhibits angiogenesis by regulating ROS-mediated apoptosis and the PI3K/AKT/mTOR signaling pathway in mES/EB-derived endothelial-like cells. Int. J. Oncol. 43, 600-610 (2013).

10. Chen, J. P., Wu, M. S., Kuo, S. H. \& Liao, F. IL-22 negatively regulates Helicobacter pylori-induced CCL20 expression in gastric epithelial cells. PLoS ONE 9, e97350 (2014).

11. Yu, J., Zhang, L., Hwang, P. M., Kinzler, K. W. \& Vogelstein, B. PUMA induces the rapid apoptosis of colorectal cancer cells. Mol. Cell 7, 673-682 (2001).

12. Nakano, K. \& Vousden, K. H. PUMA, a novel proapoptotic gene, is induced by p53. Mol. Cell 7, 683-694 (2001).

13. $\mathrm{Yu}, \mathrm{J}$. \& Zhang, L. PUMA, a potent killer with or without p53. Oncogene $\mathbf{2 7}$ (Suppl 1), S71-S83 (2008).

14. Ming, L., Wang, P., Bank, A., Yu, J. \& Zhang, L. PUMA dissociates Bax and BCl-X(L) to induce apoptosis in colon cancer cells. J. Biol. Chem. 281, 16034-16042 (2006).

15. Yu, J., Wang, P., Ming, L., Wood, M. A. \& Zhang, L. SMAC/Diablo mediates the proapoptotic function of PUMA by regulating PUMA-induced mitochondrial events. Oncogene 26, 4189-4198 (2007).

16. Yu, J., Wang, Z., Kinzler, K. W., Vogelstein, B. \& Zhang, L. PUMA mediates the apoptotic response to p53 in colorectal cancer cells. Proc. Natl Acad. Sci. USA 100, 1931-1936 (2003)

17. Sun, J. et al. Aurora kinase inhibition induces PUMA via NF-kappaB to kill colon cancer cells. Mol. Cancer Ther. 13, 1298-1308 (2014).

18. Qiu, W. et al. PUMA-mediated intestinal epithelial apoptosis contributes to ulcerative colitis in humans and mice. J. Clin. Invest. 121, 1722-1732 (2011).

19. Wang, P. et al. PUMA is directly activated by NF-kappaB and contributes to TNF-alpha-induced apoptosis. Cell Death Differ. 16, 1192-1202 (2009).

20. Harford, T. J., Kliment, G., Shukla, G. C. \& Weyman, C. M. The muscle regulatory transcription factor MyoD participates with p53 to directly increase the expression of the pro-apoptotic Bcl2 family member PUMA. Apoptosis 22, 1532-1542 (2017).

21. Fyfe, J. C. et al. The functional cobalamin (vitamin B12)-intrinsic factor receptor is a novel complex of cubilin and amnionless. Blood 103, 1573-1579 (2004).

22. Maguire, O., O'Loughlin, K. \& Minderman, H. Simultaneous assessment of NFkappaB/p65 phosphorylation and nuclear localization using imaging flow cytometry. J. Immunol. Methods 423, 3-11 (2015).
23. Obonyo, M. et al. Deficiencies of myeloid differentiation factor 88, Toll-like receptor 2 (TLR2), or TLR4 produce specific defects in macrophage cytokine secretion induced by Helicobacter pylori. Infect. Immun. 75, 2408-2414 (2007)

24. Zhang, S. et al. Early or late antibiotic intervention prevents Helicobacter pyloriinduced gastric cancer in a mouse model. Cancer Lett. 355, 106-112 (2014).

25. Xia, H. H. \& Talley, N. J. Apoptosis in gastric epithelium induced by Helicobacter pylori infection: implications in gastric carcinogenesis. Am. J. Gastroenterol. 96, 16-26 (2001)

26. Bauer, A. et al. The NF-kappaB regulator $\mathrm{BCl}-3$ and the $\mathrm{BH} 3$-only proteins Bim and Puma control the death of activated T cells. Proc. Natl Acad. Sci. USA 103 10979-10984 (2006)

27. Ina, K et al. Resistance of Crohn's disease T cells to multiple apoptotic signals is associated with a BCl-2/Bax mucosal imbalance. J. Immunol. 163, 1081-1090 (1999).

28. Verstege, M. I., te Velde, A. A. \& Hommes, D. W. Apoptosis as a therapeutic paradigm in inflammatory bowel diseases. Acta Gastroenterol. Belg. 69, 406-412 (2006).

29. Lamb, A. \& Chen, L. F. The many roads traveled by Helicobacter pylori to NFkappaB activation. Gut Microbes 1, 109-113 (2010).

30. Maeda, S. et al. Distinct mechanism of Helicobacter pylori-mediated NF-kappa B activation between gastric cancer cells and monocytic cells. J. Biol. Chem. 276 44856-44864 (2001)

31. Kawahara, T. et al. Toll-like receptor 4 regulates gastric pit cell responses to Helicobacter pylori infection. J. Med. Invest. 48, 190-197 (2001).

32. Leibowitz, B. J. et al. Targeting p53-dependent stem cell loss for intestinal chemoprotection. Sci. Transl. Med. https://doi.org/10.1126/scitranslmed. aam7610 (2018)

33. Durai, P. et al. Toll-like receptor 2 antagonists identified through virtual screening and experimental validation. FEBS J. 284, 2264-2283 (2017).

34. Wen, J. et al. Helicobacter pylori infection promotes aquaporin 3 expression via the ROS-HIF-1a-AQP3-ROS loop in stomach mucosa: a potential novel mechanism for cancer pathogenesis. Oncogene 37, 3549-3561 (2018).

35. Qiu, W. et al. PUMA regulates intestinal progenitor cell radiosensitivity and gastrointestinal syndrome. Cell Stem Cell 2, 576-583 (2008).

36. Wu, B. et al. p53 independent induction of PUMA mediates intestinal apoptosis in response to ischaemia-reperfusion. Gut 56, 645-654 (2007).

37. Yin, X. M. et al. Bid-deficient mice are resistant to Fas-induced hepatocellular apoptosis. Nature 400, 886-891 (1999).

38. Rugge, M. \& Genta, R. M. Staging and grading of chronic gastritis. Hum. Pathol. 36, 228-233 (2005). 